\title{
Anemia preoperatoria en la cirugía coronaria: ¿un factor de riesgo?
}

\author{
M. Riera ${ }^{a}, *$, J. Ibáñez ${ }^{a}$, M. Molina ${ }^{a}$, J.I. Sáez de lbarra ${ }^{b}$, J. Herrero ${ }^{a}$, A. Carrillo ${ }^{a}$, \\ C. Campillo y O. Bonnín
}

aservicio de Medicina Intensiva, Hospital Son Dureta, Palma de Mallorca, España
bServicio de Cirugía Cardíaca, Hospital Son Dureta, Palma de Mallorca, España
cInstitut Balear de la Salut, Hospital Son Dureta, Palma de Mallorca, España

Recibido el 17 de febrero de 2009; aceptado el 20 de abril de 2009

Disponible en Internet el 4 de octubre de 2009

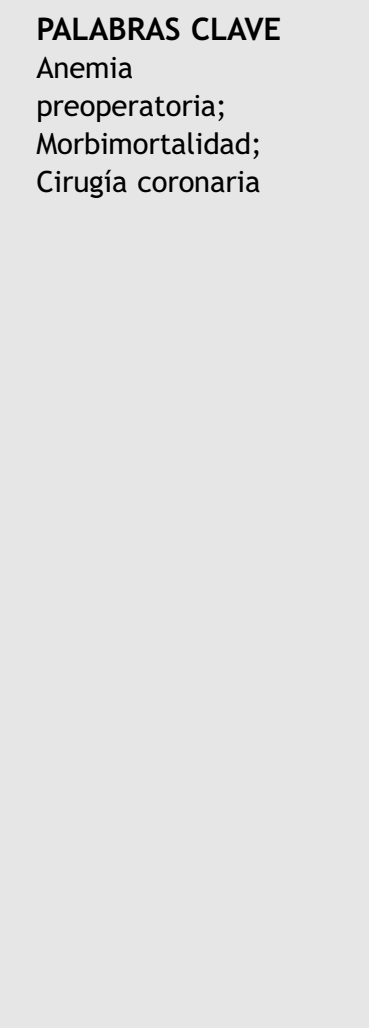

\begin{abstract}
Resumen
Objetivo: El papel de la anemia preoperatoria como factor de riesgo en la cirugía coronaria no está bien establecido. El objetivo de este estudio es analizar la asociación de la anemia preoperatoria con las complicaciones postoperatorias y la mortalidad hospitalaria en los enfermos operados de cirugía coronaria aislada en el Hospital de Son Dureta.

Material y métodos: Se incluyeron en este estudio todos los pacientes operados de cirugía coronaria aislada con circulación extracorpórea desde noviembre de 2002 hasta junio de 2007. La anemia preoperatoria se definió según los criterios de la OMS como una hemoglobina inferior a $13 \mathrm{~g} / \mathrm{dl}$ en los hombres e inferior a $12 \mathrm{~g} / \mathrm{dl}$ en las mujeres. Los efectos adversos postoperatorios cardíacos y no cardíacos se analizaron en función de la presencia o ausencia de la anemia preoperatoria y de la estratificación del riesgo quirúrgico, sobre la base del valor del EuroSCORE logístico.

Resultados: Se incluyeron 623 pacientes. La incidencia de anemia preoperatoria fue del $34,5 \%$. Esta incidencia fue mayor en pacientes con EuroSCORE de 4 o más que con EuroSCORE inferior a 4 (el 41 y el $27 \%$; $p=0,0001$ ). No hubo diferencias significativas entre la incidencia de complicaciones postoperatorias de los pacientes con anemia y sin anemia. La estancia media en la UCl y hospitalaria fue más larga en los pacientes con anemia preoperatoria que en los pacientes sin ella ( $\mathrm{UCl}: 3,2 \pm 2,5$ días y $3,7 \pm 2,8 ; \mathrm{p}=0,004$; hospitalaria: $17,5 \pm 11,3$ días y $14,7 \pm 10,2 ; \mathrm{p}=0,001)$. La mortalidad hospitalaria fue del $0,8 \%$ (intervalo de confianza del $95 \%: 0,3-1,9$ ). No hubo diferencias en la mortalidad de los pacientes con anemia preoperatoria y sin anemia preoperatoria (el 0,9 y el $0,7 \% ; p=0,8$ ). Conclusiones: En este estudio, la anemia preoperatoria en los pacientes operados de cirugía coronaria aislada no se asoció con una mayor morbimortalidad hospitalaria, aunque la estancia hospitalaria fue más larga. La limitación del tamaño de la muestra impide confirmar que la anemia preoperatoria sea o no un factor de riesgo en la cirugía coronaria. (c) 2009 Elsevier España, S.L. y SEMICYUC. Todos los derechos reservados.
\end{abstract}

*Autor para correspondencia.

Correo electrónico: rierasagrera@gmail.com (M. Riera). 


\section{KEYWORDS}

Preoperative anemia;

Mortality;

Morbidity;

Coronary artery

bypass graft surgery

\section{Preoperative anemia in coronary surgery: A risk factor?}

\begin{abstract}
Introduction and objectives: Preoperative anemia as a risk factor of adverse outcomes after coronary surgery has not been well-established. This study has aimed to analyze the association between preoperative anemia and postoperative adverse events and inhospital mortality in the patients undergoing isolated coronary artery bypass graft surgery in the Son Dureta hospital.

Methods: All the patients undergoing isolated coronary artery bypass graft surgery with extracorporeal circulation from November 2002 to June 2007 were included. Preoperative anemia was defined as hemoglobin $(\mathrm{Hb})<13 \mathrm{~g} / \mathrm{dL}$ in men and $\mathrm{Hb}<12 \mathrm{~g} / \mathrm{dL}$ in women. The association between postoperative cardiac and noncardiac adverse events and the presence or absence of preoperative anemia and concomitant surgical risk, assessed by logistic EuroScore, were analyzed.

Results: A total of 623 patients were included. The rate of preoperative anemia was $34.5 \%$. Patients with Euroscore $\geq 4$ had higher incidence of preoperative anemia than patients with Euroscore $<4(41 \%$ vs. $27 \% ; p=0.0001)$. There were no statistically significant differences in the rate of postoperative adverse events related to the presence or absence of preoperative anemia. Median ICU and hospital length of stay were longer in patients with preoperative anemia than in patients without preoperative anemia (ICU: $3.2 \pm 2.5$ days vs. $3.7 \pm 2.8, p=0.004$; inhospital: $17.5 \pm 11.3$ days vs. $14.7 \pm 10.2, p=0.001)$. Hospital mortality rate was $0.8 \%(95 \% \mathrm{Cl} 0.3-1.9)$. There were no differences in the mortality rate of the patients with and without preoperative anemia ( $0.9 \%$ vs $0.7 \%, \mathrm{p}=0.8)$.

Conclusions: In this study, preoperative anemia in patients undergoing coronary artery bypass graft surgery was not associated with increased hospital morbidity-mortality. However, ICU and hospital length of stay were longer in patients with preoperative anemia. The limitation of the sample size prevents us from confirming whether preoperative anemia is a risk factor after coronary surgery or not.

(c) 2009 Elsevier España, S.L. and SEMICYUC. All rights reserved.
\end{abstract}

La anemia preoperatoria es un hallazgo frecuente en los pacientes candidatos a cirugía coronaria ${ }^{1}$. El estudio de la anemia preoperatoria como un factor de riesgo independiente de la morbimortalidad en los pacientes operados de cirugía coronaria muestra resultados discordantes ya que, mientras unos estudios demuestran que es un factor asociado a una mayor morbimortalidad ${ }^{1,2}$, otros no lo confirman $^{3}$. Conocer la repercusión de la anemia preoperatoria en la cirugía cardíaca es interesante porque la anemia es susceptible de corregirse antes del acto operatorio. Determinar si la corrección de la anemia es beneficiosa para los enfermos operados de cirugía cardíaca con circulación extracorpórea (CEC) es uno de los objetivos de un ensayo clínico todavía no finalizado ${ }^{4}$.

La morbimortalidad secundaria a la cirugía coronaria ha ido disminuyendo debido a las mejoras de la técnica quirúrgica y al cuidado postoperatorio, a pesar de que los pacientes operados en la actualidad presentan un perfil de riesgo operatorio elevado porque su edad es mucho mayor y tienen mayor número de enfermedades asociadas ${ }^{5}$. Aun así, la mortalidad de la cirugía coronaria aislada y el análisis de los factores de riesgo relacionados con el pronóstico en este tipo de cirugía muestran notables diferencias ${ }^{6-8}$ en artículos publicados recientemente.

Ante los resultados discrepantes que muestra la literatura médica, nuestro objetivo fue examinar la asociación de la anemia preoperatoria con los efectos adversos postoperatorios y la mortalidad hospitalaria en los pacientes operados de cirugía coronaria aislada.

\section{Pacientes y métodos}

\section{Población de estudio y diseño}

Se trata de un estudio que incluye a todos los pacientes operados de cirugía coronaria aislada con CEC en el Hospital Universitario Son Dureta de Palma de Mallorca desde el inicio de la actividad de cirugía cardíaca, en noviembre de 2002, hasta el 30 de junio de 2007. Nuestro hospital es el centro de referencia para la cirugía cardíaca de la comunidad de las Islas Baleares y cubre la asistencia de una población cercana al millón de habitantes.

Se intervino a todos los pacientes mediante una esternotomía media y CEC. Se utilizó cardioplejía cristaloide fría. Durante la operación se monitorizó sistemáticamente la protección miocárdica y se midió de forma continua la temperatura septal. Al salir de la CEC, se comprobó sistemáticamente la permeabilidad de los injertos y se calculó el índice de permeabilidad mediante ultrasonidos intraoperatorios (Transonic Systems Inc, Ithaca, NY, EE. UU.).

Los pacientes ingresaron en la $\mathrm{UCl}$ sedados y conectados a ventilación mecánica y se extubaron una vez despiertos y estables, según un protocolo establecido. Durante la 
intervención quirúrgica se transfundieron concentrados de hematíes a los pacientes menores de 70 años con hemoglobina $(\mathrm{Hb})$ inferior a $8 \mathrm{~g} / \mathrm{dl}$ y a los mayores de 70 años con $\mathrm{Hb}$ inferior a $9 \mathrm{~g} / \mathrm{dl}$. Durante la estancia en la $\mathrm{UCl}$ se transfundieron los pacientes con $\mathrm{Hb}$ inferior a $8 \mathrm{~g} / \mathrm{dl}$, aquéllos con $\mathrm{Hb}$ inferior a $10 \mathrm{~g} / \mathrm{dl}$ y con daño neurológico o miocárdico, y aquéllos en que se apreciaban signos de hemorragia activa grave.

\section{Recogida de datos y definiciones}

Los intensivistas y los cirujanos cardíacos introdujeron los datos de todos los pacientes operados de cirugía coronaria en una base de datos clínica (Microsoft Access, Microsoft, Redmond, WA) de manera prospectiva. Se recogieron los datos perioperatorios (incluyendo los demográficos, las comorbilidades previas a la cirugía, los resultados de laboratorio, el tipo de cirugía, las transfusiones de productos sanguíneos durante la operación y en la $\mathrm{UCl}$, los efectos adversos postoperatorios, el tiempo de ventilación mecánica y el tiempo de estancia en la UCI y en el hospital) a partir de la historia clínica. Se calculó la puntuación del EuroSCORE logístico9. Las complicaciones cardíacas que se analizaron durante el período postoperatorio en este estudio fueron el paro cardíaco, la fibrilación ventricular, el shock cardiogénico y el infarto agudo de miocardio. Se definió el infarto agudo de miocardio ante la presencia de nuevas ondas $Q$ o alteraciones típicas de isquemia aguda en el ECG y de un valor de la fracción MB de la creatincinasa (CK-MB) superior a 5 veces el límite superior de la normalidad ${ }^{10}$. Las complicaciones no cardíacas incluyeron el accidente cerebro vascular (ACV) agudo, la disfunción renal y las infecciones respiratorias. Se definió el ACV agudo como la aparición de un déficit focal neurológico mantenido durante al menos $24 \mathrm{~h}$ y confirmado mediante una TAC. Los criterios para el diagnóstico de mediastinitis y de neumonía se establecieron conforme a la guía publicada por el CDC. Debido a la existencia de distintas definiciones de insuficiencia renal postoperatoria en los enfermos operados de cirugía cardíaca y con el fin de poder comparar los resultados se escogió la propuesta por Kulier et $\mathrm{al}^{1}$ : el valor de creatinina sérica debía ser de $2,0 \mathrm{mg} / \mathrm{dl}$ o más y debía acompañarse de un incremento de $0,7 \mathrm{mg} / \mathrm{dl}$ o más respecto al valor basal. La anemia preoperatoria se definió según los criterios propuestos por la OMS: Hb inferior a $13 \mathrm{~g} / \mathrm{dl}$ en los hombres y $\mathrm{Hb}$ inferior a $12 \mathrm{~g} / \mathrm{dl}$ en las mujeres.

El tiempo de ventilación mecánica se definió como el período durante el cual el paciente necesitó soporte ventilatorio tras la cirugía cardíaca, desde el ingreso en la UCI hasta la extubación, incluido el tiempo de ventilación mecánica asociado a las reintubaciones. El tiempo de estancia hospitalaria incluyó el tiempo transcurrido desde la cirugía cardíaca hasta el alta hospitalaria y el tiempo de estancia en la $\mathrm{UCl}$, la duración del ingreso en la UCl tras la cirugía cardíaca, incluyendo los reingresos. La mortalidad se refirió a los pacientes que fallecieron durante la estancia hospitalaria.

\section{Análisis estadístico}

Los resultados de las variables continuas se presentan como media \pm desviación estándar o mediana (rango intercuartílico) cuando la distribución no era normal. Las variables categóricas aparecen en valor absoluto o porcentajes. Se realizó un análisis univariado para determinar el efecto de la anemia preoperatoria sobre la morbimortalidad. Para determinar la influencia de las enfermedades asociadas sobre los efectos adversos postoperatorios, el riesgo preoperatorio se valoró con el EuroSCORE logístico. La mediana de la población de estudio se utilizó como el valor de EuroSCORE que definió 2 grupos de riesgo quirúrgico: bajo riesgo quirúrgico (EuroSCORE logístico $<4$ ) y alto riesgo quirúrgico (EuroSCORE $\geq 4$ ). Las medias se compararon mediante la prueba del test $\mathrm{t}$ de Student o la prueba no paramétrica $U$ de Mann-Whitney si no seguían una distribución normal, y los porcentajes se compararon con las pruebas de $\chi^{2}$ de Pearson o exacta de Fisher. Se utilizó la regresión logística para determinar la asociación de la mortalidad con los valores de $\mathrm{Hb}$ preoperatoria y otros factores de riesgo conocidos por su repercusión sobre la mortalidad que fueron estadísticamente significativos en el análisis univariado. Se estudió también la asociación entre las variables preoperatorias asociadas a la anemia preoperatoria. Los resultados se presentan como odds ratios (OR) con sus intervalos de confianza (IC) del 95\%. Se consideró estadísticamente significativo un valor de $\mathrm{p}<0,05$. El análisis estadístico se realizó con el programa SPSS (versión 15.0, SPSS, Inc, Chicago, IL, EE. UU.).

\section{Resultados}

La tabla 1 muestra las características principales de los 623 pacientes operados de cirugía coronaria aislada. La incidencia de anemia preoperatoria fue del $34,5 \%$. Presentó anemia preoperatoria el $34,9 \%$ de los varones (172 de 493) y el 33,1\% de las mujeres (43 de 130). Los pacientes con anemia preoperatoria (tabla 1) presentaron mayor edad, mayor incidencia de diabetes mellitus no dependiente de la insulina, historia de insuficiencia renal crónica, mayor uso de clopidogrel preoperatorio y un EuroSCORE logístico más alto. Las cifras basales de creatinina y de $\mathrm{Hb}$ también fueron estadísticamente diferentes entre los pacientes con anemia y sin anemia. Los pacientes con anemia preoperatoria recibieron un mayor número de concentrados de hematíes durante la cirugía o los 3 primeros días de estancia en la $\mathrm{UCl}$. El tiempo de ventilación mecánica fue de 9,4 $\pm 21,2 \mathrm{~h}$ (mediana de $5 \mathrm{~h}$ ) y fue igual con anemia preoperatoria y sin anemia preoperatoria. En todos los pacientes se utilizó, al menos, un injerto de la arteria mamaria interna. La arteria mamaria interna se utilizó como injerto de elección a la arteria coronaria descendente anterior en el $97 \%$ de los casos. La arteria radial se utilizó como injerto en un 16\% de los pacientes. El 76\% de los pacientes recibió 3 o más injertos coronarios y no hubo diferencias asociadas con la presencia de anemia preoperatoria.

\section{Morbimortalidad}

La tabla 2 muestra que no hubo diferencias significativas entre la frecuencia de complicaciones postoperatorias cardíacas o no cardíacas en relación con la presencia o ausencia de anemia preoperatoria. La complicación 
Tabla 1 Características clínicas

\begin{tabular}{|c|c|c|c|c|}
\hline & Total & No anémicos & Anémicos & $\mathrm{p}$ \\
\hline & $(n=623)$ & $(n=408)$ & $(n=215)$ & \\
\hline Sexo femenino, n (\%) & $130(21)$ & $87(21)$ & $43(20)$ & 0,76 \\
\hline Edad, años & $63,6 \pm 10,0$ & $62 \pm 10$ & $66 \pm 9$ & $<0,0001$ \\
\hline Edad > 75 años, n (\%) & $78(13)$ & $38(9)$ & 40 (19) & 0,001 \\
\hline Peso en $\mathrm{kg}$, media \pm SD & $78,3 \pm 13,5$ & $79,4 \pm 13$ & $76,5 \pm 14$ & 0,02 \\
\hline $\mathrm{BMI}$, media $\pm \mathrm{SD}$ & $28,6 \pm 4,3$ & $29 \pm \overline{4}$ & $28 \pm \overline{4}$ & 0,06 \\
\hline Fumador, n (\%) & $156(25)$ & $103(25)$ & $53(25)$ & 0,87 \\
\hline Diabetes mellitus ID, $\mathrm{n}(\%)$ & $75(12)$ & $50(12)$ & $25(12)$ & 0,82 \\
\hline Diabetes mellitus NID, $\mathrm{n}(\%)$ & $183(29)$ & $106(26)$ & $77(36)$ & 0,01 \\
\hline Hipertensión arterial, n (\%) & $405(65)$ & $267(65)$ & $138(64)$ & 0,76 \\
\hline EPOC, $n(\%)$ & $81(13)$ & $51(12)$ & $30(14)$ & 0,61 \\
\hline Ictus, n (\%) & $51(8)$ & $33(8)$ & $18(8)$ & 0,90 \\
\hline Vasculopatía periférica, n (\%) & $76(12,2)$ & $43(10)$ & $33(15)$ & 0,08 \\
\hline Angor, n (\%) & $428(69)$ & $285(70)$ & $143(66)$ & 0,39 \\
\hline Infarto de miocardio, $\mathrm{n}(\%)$ & $313(50)$ & $198(48)$ & $115(53)$ & 0,24 \\
\hline Enfermedad del TCI, n (\%) & $162(26)$ & $98(24)$ & $64(30)$ & 0,12 \\
\hline Fracción de eyección del VI <30\%, n (\%) & $21(3)$ & $13(3)$ & $8(4)$ & 0,72 \\
\hline Insuficiencia renal crónica, n (\%) & $46(7)$ & $14(3)$ & $32(15)$ & $<0,0001$ \\
\hline Creatinina basal, media \pm SD & $1,1 \pm 0,5$ & $1 \pm 0,4$ & $1,2 \pm 0,7$ & 0,000 \\
\hline Aspirina preoperatoria, $\mathrm{n}(\%)$ & $517(83)$ & $341(84)$ & $176(82)$ & 0,59 \\
\hline Clopidogrel preoperatorio, $\mathrm{n}(\%)$ & $185(30)$ & $110(27)$ & $75(35)$ & 0,04 \\
\hline Cirugía urgente, $\mathrm{n}(\%)$ & $90(14)$ & $51(13)$ & $39(18)$ & 0,06 \\
\hline EuroSCORE logístico, media $\pm S D$ & $4,9 \pm 5,2$ & $4,3 \pm 5$ & $6,0 \pm 6$ & $<0,0001$ \\
\hline Tiempo de la CEC, min, media \pm SD & $89,3 \pm 29,3$ & $91 \pm 30$ & $85 \pm 26$ & 0,005 \\
\hline Tiempo de la isquemia, min, media \pm SD & $55 \pm 20,6$ & $56 \pm 20$ & $54 \pm 18$ & 0,22 \\
\hline N. ${ }^{\circ}$ de injertos, mediana (RIC) & $3(2-3)$ & $3(\overline{1}-5)$ & $3(1-5)$ & 0,82 \\
\hline $\mathrm{Hb}$ basal en $\mathrm{g} / \mathrm{dl}$, media $\pm \mathrm{SD}$ & $13,2 \pm 1,6$ & $14,1 \pm 1,1$ & $11,6 \pm 1,1$ & $<0,0001$ \\
\hline Concentrados de hematies*, mediana (RIC) & $2(0-3)$ & $2(0-15)$ & $3(0-13)$ & $<0,0001$ \\
\hline $0, \mathrm{n}(\%)$ & $187(30)$ & $158(39)$ & $29(14)$ & \\
\hline $1-4, \mathrm{n}(\%)$ & $367(59)$ & $222(54)$ & $145(67)$ & \\
\hline $5-8, \mathrm{n}(\%)$ & $60(10)$ & $22(5)$ & $38(18)$ & \\
\hline$>8, \mathrm{n}(\%)$ & $9(1)$ & $6(2)$ & $3(1)$ & \\
\hline
\end{tabular}

BMI: índice de masa corporal; CEC: circulación extracorpórea; EPOC: enfermedad pulmonar obstructiva crónica; Hg: hemoglobina; ID: dependiente de la insulina; NID: no dependiente de la insulina; RIC: rango intercuartílico; TCI: tronco común izquierdo; VI: ventrículo izquierdo.

$* n=622$.

Tabla 2 Incidencia de complicaciones postoperatorias

\begin{tabular}{|c|c|c|c|c|}
\hline & Total & No anémicos & Anémicos & $\mathrm{p}$ \\
\hline & $(n=623)$ & $(n=408)$ & $(n=215)$ & \\
\hline Ventilación mecánica > 72 h, n (\%) & $6(1)$ & $3(1)$ & $3(1)$ & 0,42 \\
\hline Reintubación, n (\%) & $11(2)$ & $8(2)$ & $3(1)$ & 0,61 \\
\hline Infarto agudo miocardio, n (\%) & $27(4)$ & $15(4)$ & $12(6)$ & 0,23 \\
\hline Fibrilacion ventricular, n (\%) & $9(1,5)$ & $5(1,2)$ & $4(1,8)$ & 0,54 \\
\hline Shock cardiogénico, n (\%) & $7(1,1)$ & $5(1,2)$ & $2(0,9)$ & 0,73 \\
\hline Insuficiencia renal aguda, n (\%) & $22(3,5)$ & $11(2,7)$ & $11(5,1)$ & 0,12 \\
\hline Ictus isquémico o hemorrágico, $\mathrm{n}$ (\%) & $7(1,1)$ & $5(1,2)$ & $2(0,9)$ & 0,73 \\
\hline Infección respiratoria, $\mathrm{n}(\%)$ & $9(1,5)$ & $5(1,2)$ & $4(1,8)$ & 0,51 \\
\hline
\end{tabular}


Tabla 3 Complicaciones postoperatorias según el EuroSCORE

\begin{tabular}{|c|c|c|c|c|}
\hline & Todos los pacientes & EuroSCORE $<4$ & EuroSCORE $\geq 4$ & $\mathrm{p}$ \\
\hline & $n=623$ & $\mathrm{n}=289$ & $n=334$ & \\
\hline \multicolumn{5}{|l|}{ Cardíacas } \\
\hline Fibrilación ventricular, n (\%) & $9(1,5)$ & $1(0,3)$ & $8(2,3)$ & 0,03 \\
\hline IAM, n (\%) & $27(4,3)$ & $8(2,8)$ & $19(5,7)$ & 0,07 \\
\hline PCR, n (\%) & $9(1,3)$ & $4(1,2)$ & $5(1,4)$ & 0,052 \\
\hline Shock cardiogénico, n (\%) & $7(1,1)$ & $0(0)$ & $7(2,1)$ & 0,01 \\
\hline \multicolumn{5}{|l|}{ No cardíacas } \\
\hline Infección respiratoria, n (\%) & $9(1,5)$ & $2(0,7)$ & $7(2,1)$ & 0,31 \\
\hline Reintubación, n (\%) & $11(1,8)$ & $1(0,3)$ & $10(3)$ & 0,01 \\
\hline Ictus, n (\%) & $7(1,2)$ & $1(0,3)$ & $6(1,8)$ & 0,14 \\
\hline Insuficiencia renal aguda, n (\%) & $22(3,5)$ & $5(1,7)$ & $17(5)$ & 0,04 \\
\hline
\end{tabular}

IAM: infarto agudo de miocardio; PCR: parada cardiorrespiratoria.

postoperatoria grave más frecuente fue el infarto agudo de miocardio, seguida por la insuficiencia renal aguda. Entre las complicaciones infecciosas respiratorias, un paciente desarrolló neumonía y 13 pacientes traqueobronquitis. La reintervención quirúrgica se realizó en 9 de 623 pacientes $(1,4 \%)$. Los pacientes con mayor riesgo quirúrgico (EuroSCORE $\geq 4$ ) presentaron una mayor frecuencia de shock cardiogénico, reintubación e insuficiencia renal aguda (tabla 3) y una mayor incidencia de anemia preoperatoria (el 41 y el $27 \%$; $p<0,0001$ ).

La estancia media en la UCl fue de $3,2 \pm 2,5$ días (mediana de 2 días) en los pacientes sin anemia preoperatoria y de $3,7 \pm 2,8$ (mediana de 3 días) en aquellos con anemia preoperatoria $(p=0,004)$. La estancia media en el hospital también fue más larga en los pacientes con anemia preoperatoria que en los que no la tenían $(17,5 \pm 11,3$ días; mediana de 14 días y 14,7 $\pm 10,2$; mediana de 12 días; $\mathrm{p}=0,001)$. La mortalidad hospitalaria observada fue del $0,8 \%$ (IC del 95\%: 0,3-1,9). No hubo diferencias entre la mortalidad de los pacientes con anemia preoperatoria y sin anemia preoperatoria (el 0,9 y el $0,7 \% ; \mathrm{p}=0,79$ ). La mortalidad esperada, según el EuroSCORE logístico, fue del 4,9\%.

Los 26 pacientes con anemia preoperatoria grave $(\mathrm{Hb}<10$ $\mathrm{g} / \mathrm{dl}$ ) presentaron una mortalidad del $3,8 \%$ respecto al $0,7 \%$ del resto de los pacientes con $\mathrm{Hb}$ de $10 \mathrm{~g} / \mathrm{dl}$ o más $(\mathrm{p}=0,8)$. Estos pacientes eran de mayor edad y tenían mayor riesgo quirúrgico según el EuroSCORE que los pacientes sin anemia preoperatoria.

En el análisis de regresión logística no se encontró ninguna asociación entre la mortalidad y la anemia preoperatoria. Este análisis indicó que hubo 2 factores asociados con la anemia preoperatoria: la historia de insuficiencia renal crónica $(\mathrm{OR}=3,02$; IC del 95\%: 1,47-6,30) y la edad $(\mathrm{OR}=1,04$; IC del 95\%: 1,02-1,06).

\section{Discusión}

En este estudio, los pacientes operados de cirugía coronaria aislada que presentaban anemia preoperatoria tuvieron complicaciones postoperatorias similares al grupo de pacientes sin anemia, aunque su estancia hospitalaria fue más larga. La mortalidad hospitalaria también fue similar entre ambos grupos.

La incidencia de anemia preoperatoria moderada detectada en este estudio fue comparable a la de otros artículos $^{1,11}$ y se asoció a la población de mayor edad, la historia de insuficiencia renal crónica y al mayor riesgo quirúrgico. La incidencia de anemia preoperatoria grave $(\mathrm{Hb}<10 \%)$ fue del $4 \%$, muy similar a la observada en otros estudios $^{1-3}$.

Kulier et $\mathrm{al}^{1}$ demostraron que la anemia preoperatoria, que cursa con valores de $\mathrm{Hb}$ inferior a $11 \mathrm{~g} / \mathrm{dl}$, se asocia significativamente con la aparición de complicaciones postoperatorias no cardíacas (especialmente con la lesión renal) y aumenta la estancia hospitalaria. Aunque la frecuencia de insuficiencia renal postoperatoria fue similar a la encontrada por nosotros, no detectamos una asociación significativa entre la anemia preoperatoria y la aparición de complicaciones postoperatorias. Sin embargo, estos autores tampoco encontraron una asociación significativa de la anemia preoperatoria con las complicaciones cardíacas. En un estudio muy reciente, Bell et $\mathrm{al}^{3}$ estratificaron a los pacientes operados de cirugía coronaria en 3 grupos (con anemia preoperatoria grave, moderada y sin anemia) y no pudieron confirmar la asociación de la anemia preoperatoria con una mayor morbilidad postoperatoria, lo que coincide con nuestros resultados.

Los datos discrepantes pueden deberse, en parte, a diferencias metodológicas. Cada estudio incluyó variables distintas para el ajuste estadístico; las definiciones empleadas (por ejemplo, para la anemia, la insuficiencia renal o el accidente vascular cerebral) no fueron las mismas y no todos disponían de información relacionada con la transfusión realizada. Del mismo modo, existían diferencias con la frecuencia de algunas enfermedades asociadas observadas en nuestra serie respecto al estudio de Kulier et $\mathrm{al}^{1}$ : mayor frecuencia de pacientes con diabetes y menor frecuencia de pacientes con enfermedad renal crónica. En nuestro estudio, las complicaciones más frecuentes, después de la cirugía coronaria, fueron el infarto agudo de miocardio y la insuficiencia renal aguda, aunque no hubo diferencias significativas entre los pacientes con anemia preoperatoria y $\sin$ anemia preoperatoria. 
Tabla 4 Mortalidad hospitalaria en la cirugía coronaria aislada con circulación extracorpórea

\begin{tabular}{lll}
\hline Estudios & Pacientes & Mortalidad \\
\cline { 2 - 3 } & (n) & (\%) \\
\hline Nuestro estudio & 623 & 0,8 \\
Bell $^{3+} 2008$ & 36.658 & 3,1 \\
Lafuente $^{6} 2008$ & 498 & 5,8 \\
Sobolev $^{8} 2008$ & 7.316 & 1,3 \\
Kulier $^{1} 2007$ & 4.804 & 3,3 \\
Cislaghi $^{23} 2007$ & 3.269 & 3 \\
Ribera $^{7} 2006$ & 1.267 & 5,1 \\
\hline
\end{tabular}

Cirugía coronaria programada: $\uparrow$ mortalidad a 30 días.

Kulier et $\mathrm{al}^{1}$ utilizaron como objetivo principal una variable combinada que dificulta la comparación con nuestros resultados. Además, presentaron los datos de mortalidad estratificados por niveles de $\mathrm{Hb}$, pero no publicaron la mortalidad global de los pacientes con anemia o sin anemia, lo que dificulta la comparación con nuestra serie. El uso de las variables combinadas en los ensayos clínicos y en estudios retrospectivos es cada vez más frecuente $^{12}$, engloba en una única variable diferentes resultados e incluye al menos una de las complicaciones seleccionadas. Es necesario que dicha variable combinada cumpla con una serie de requisitos, entre los que hay que destacar que los componentes individuales tengan una importancia clínica similar para el paciente y sean biológicamente plausibles. Se recomienda que todos los componentes de una variable compuesta se analicen separadamente para detectar si un tratamiento afecta a todos los componentes o sólo a un componente aislado ${ }^{13}$.

La diferencia entre la mortalidad de los pacientes operados de cirugía coronaria con anemia preoperatoria y sin anemia preoperatoria moderada no fue estadísticamente significativa, lo que coincide con los 2 estudios citados ${ }^{1,3}$. Los pacientes con anemia preoperatoria grave $(\mathrm{Hb} \leq 10 \mathrm{~g} / \mathrm{dl})$ tuvieron una mortalidad del 3,8\%, muy similar a la observada por Bell et $\mathrm{al}^{3}$, pero inferior a la encontrada en otros $^{1,2,11}$. La mortalidad hospitalaria de la cirugía coronaria aislada ha ido disminuyendo progresivamente en esta última década $^{5}$, aunque estudios recientes (tabla 4) muestran todavía importantes diferencias entre centros y países, que son explicables en parte porque el tipo de los pacientes estudiados, la indicación de cirugía urgente o emergente y los procesos de cuidados clínicos aplicados no son siempre similares. La mejora en la aplicación de los distintos procesos de cuidados clínicos de la cirugía coronaria ha contribuido a mejorar los resultados no sólo de la mortalidad $^{14}$, sino de otros parámetros de mejor calidad asistencial (por ejemplo, la frecuencia de uso del injerto de la arteria mamaria interna en pacientes mayores de 75 años $\left.{ }^{15}\right)$.

¿Debemos trasfundir antes de la cirugía coronaria a los pacientes con anemia preoperatoria? No hay estudios publicados que demuestren el efecto de esta estrategia, por lo que, en principio, la recomendación es no cambiar las actuales normas de transfusión de concentrados de hematíes. Se ha descrito que la transfusión de hematíes durante la cirugía cardíaca puede aumentar el número de infecciones graves, especialmente la neumonía ${ }^{16}$, y que se asocia con una disminución de la supervivencia hospitalaria y a largo plazo ${ }^{17-19}$. Sin embargo, en estos estudios es difícil averiguar si el factor relevante es la transfusión per se o la complicación que condicionó la anemia y la posterior transfusión. Se desconocen las posibles causas de esta anemia, conocerlas podría ser importante para determinar posibles tratamientos preoperatorios. La interacción de la insuficiencia renal crónica con la anemia podría aumentar el riesgo quirúrgico de la cirugía cardíaca, aunque en un estudio reciente en pacientes operados de cirugía valvular aislada o combinada con cirugía coronaria no pudimos demostrar este efecto ${ }^{20}$.

Entre las limitaciones de este estudio debemos destacar el reducido número de pacientes si tenemos en cuenta a los 3 estudios más semejantes al nuestro, que incluyeron entre 2.059 y 36.658 pacientes $^{1-3}$. El número absoluto de las complicaciones postoperatorias observadas (muerte, insuficiencia renal aguda, shock cardiogénico, etc.) también fue pequeño, lo que no permite generar una conclusión definitiva. Recientemente se ha estudiado la fiabilidad de la mortalidad como un indicador de la calidad asistencial del hospital cuando el número de procedimientos quirúrgicos realizados es bajo y hay una baja incidencia de complicaciones ${ }^{21,22}$. Los autores llegaron a la conclusión de que la mortalidad de la cirugía coronaria es un indicador fiable para detectar la calidad del rendimiento de un hospital siempre que se opere un mínimo número de 219 pacientes al año, motivo por el que el tamaño de nuestro estudio es insuficiente para estimar, con la precisión deseada, la morbimortalidad asociada a la cirugía coronaria practicada en nuestro centro. Creemos que es necesaria una mayor investigación a nivel multicéntrico para determinar si la anemia preoperatoria es un factor de riesgo de morbimortalidad.

Como conclusión en este estudio, la anemia preoperatoria en los pacientes operados de cirugía coronaria aislada no se asoció con una mayor frecuencia de mortalidad ni de complicaciones postoperatorias, pero la estancia hospitalaria fue mayor. La limitación del tamaño de la muestra impide confirmar que la anemia preoperatoria sea o no un factor de riesgo en la cirugía coronaria.

\section{Bibliografía}

1. Kulier A, Levin J, Moser R, Rumpold-Seitlinger G, Tudor IC, Snyder-Ramos SA, et al. Impact of preoperative anemia on outcome in patients undergoing coronary artery bypass graft surgery. Circulation. 2007;116:471-9.

2. Zindrou D, Taylor KM, Bagger JP. Preoperative haemoglobin concentration and mortality rate after coronary artery bypass surgery. Lancet. 2002;359:1747-8.

3. Bell ML, Grunwald GK, Baltz JH, MCDonald GO, Bell MR, Grover $\mathrm{FL}$, et al. Does preoperative hemoglobin independently predict short-term outcomes after coronary artery bypass graft surgery?. Ann Thorac Surg. 2008;86:1415-23.

4. Clincal trials.gov, NCT00651573. Transfusion strategies in cardiac surgery. [citado 10 Feb 2009]. Disponible en: URL: http://www.clinicaltrials.gov.

5. Biancari F, Kangasniemi OP, Mahar MA, Rasinaho E, Satomaa A, Tiozzo V, et al. Changing risk of patients undergoing coronary 
artery bypass surgery. Interact Cardiovasc Thorac Surg. 2009;8:40-4.

6. Lafuente S, Trilla A, Bruni L, González R, Bertran MJ, Pomar JL, et al. Validación del modelo probabilístico EuroSCORE en pacientes intervenidos de injerto coronario. Rev Esp Cardiol. 2008;61:589-94.

7. Ribera A, Ferreira-González I, Cascant P, Pons JM, PermanyerMiralda G. Evaluación de la mortalidad hospitalaria ajustada al riesgo de la cirugía coronaria en la sanidad pública catalana. Influencia del tipo de gestión del centro (estudio ARCA). Rev Esp Cardiol. 2006;59:431-40.

8. Sobolev BG, Fradet G, Hayden R, Kuramoto L, Levy AR, FitzGerald MJ. Delay in admission for elective coronary-artery bypass grafting is associated with increased in-hospital mortality. BMC Health Serv Res. 2008;8:185.

9. Roques F, Michel P, Goldstone AR, Nashef SA. The logistic EuroSCORE. Eur Heart J. 2003;24:881-2.

10. Riera M, Carrillo A, Ibañez J, Sáez de Ibarra JI, Fiol M, Bonnín O. Valor predictivo del modelo EuroSCORE en la cirugía cardíaca de nuestro centro. Med Intensiva. 2007;31:231-6.

11. Karkouti K, Wijeysundera DN, Beattie WS. Risk associated with preoperative anemia in cardiac surgery: A multicenter cohort study. Circulation. 2008;117:478-84.

12. Ferreira-González I, Alonso-Coello P, Sola I, Pacheco-Huergo V, Domingo-Salvany A, Alonso J, et al. Variables de resultado combinadas en los ensayos clínicos. Rev Esp Cardiol. 2008;61: 283-90.

13. Ferreira-González I, Permanyer-Miralda G, Busse JW, Bryant DM, Montori VM, Alonso-Coello P, et al. Methodologic discussions for using and interpreting composite endpoints are limited, but still identify major concerns. J Clin Epidemiol. 2007;60:651-7.

14. O’Connor GT, Plume SK, Olmstead EM, Morton JR, Maloney CT, Nugent WC, et al. A regional intervention to improve the hospital mortality associated with coronary artery bypass graft surgery. The Northern New England Cardiovascular Disease Study Group. JAMA. 1996;275:841-6.

15. Ferguson Jr TB, Peterson ED, Coombs LP, Eiken MC, Carey ML, Grover FL, et al. Use of continuous quality improvement to increase use of process measures in patients undergoing coronary artery bypass graft surgery: A randomized controlled trial. JAMA. 2003;290:49-56.

16. Leal-Noval SR, Rincón-Ferrari MD, Garcia-Curiel A, HerruzoAviles A, Camacho-Larana P, Garnacho-Montero J, et al. Transfusion of blood components and postoperative infection in patients undergoing cardiac surgery. Chest. 2001;119: 1461-1468.

17. Koch CG, Li L, Duncan Al, Mihaljevic T, Loop FD, Starr NJ, et al. Transfusion in coronary artery bypass grafting is associated with reduced long-term survival. Ann Thorac Surg. 2006;81:1650-7.

18. Koch CG, Li L, Duncan Al, Mihaljevic T, Cosgrove DM, Loop FD, et al. Morbidity and mortality risk associated with red blood cell and blood-component transfusion in isolated coronary artery bypass grafting. Crit Care Med. 2006;34:1608-16.

19. Murphy GJ, Reeves BC, Rogers CA, Rizvi SI, Culliford L, Angelini $\mathrm{GD}$. Increased mortality, postoperative morbidity, and cost after red blood cell transfusion in patients having cardiac surgery. Circulation. 2007;116:2544-52.

20. Ibañez J, Riera M, Sáez de Ibarra JI, Carrillo A, Fernández R, Herrero J, et al. Effect of preoperative mild renal dysfunction on mortality and morbidity following valve cardiac surgery. Interact Cardiovasc Thorac Surg. 2007;6:748-52.

21. Dimick JB, Welch HG. The zero mortality paradox in surgery. J Am Coll Surg. 2008;206:13-16.

22. Dimick JB, Welch HG, Birkmeyer JD. Surgical mortality as an indicator of hospital quality: The problem with small sample size. JAMA. 2004;292:847-51.

23. Cislaghi F, Condemi AM, Corona A. Predictors of prolonged mechanical ventilation in a cohort of 3,269 CABG patients. Minerva Anestesiol. 2007;73:615-21. 